\title{
FUTURO DE LAS REVISTAS CIENTÍFICAS DE COMUNICACIÓN EN ESPAÑA
}

\author{
Antonio J. Baladrón-Pazos y Beatriz Correyero-Ruiz
}
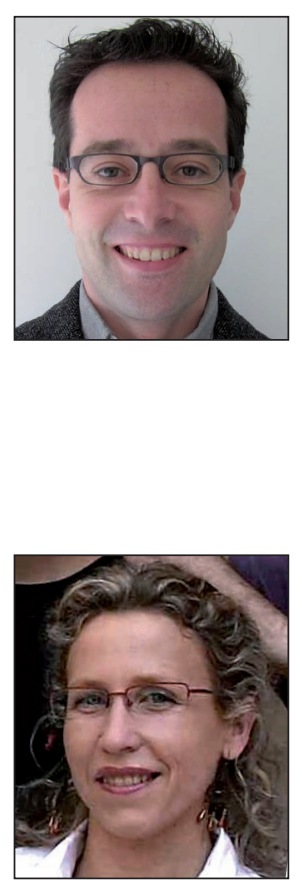

Antonio J. Baladrón-Pazos, doctor en publicidad y relaciones públicas y profesor contratado doctor de esta especialidad en el Departamento de Ciencias de la Comunicación I de la Univ. Rey Juan Carlos (URJC) de Madrid, es licenciado en periodismo y en publicidad y relaciones públicas. Ha sido docente en la Univ. Pontifica de Salamanca, la Univ. Católica San Antonio y la Univ. de Málaga. Ha participado en actividades académicas y como profesor invitado en diferentes universidades extranjeras: Univ. de Coimbra (Portugal), Univ. degli Studi di Sassari (Italia), Wroclaw Univ. (Polonia), Univ. de Antioquia (Colombia), Pontificia Univ. Católica de São Paulo (Brasil) y Univ. Autónoma de Yucatán (México). Participa en varios proyectos de I+D financiados por el Ministerio de Ciencia e Innovación y por la Comunidad de Madrid.

Universidad Rey Juan Carlos Edif. Departamental I, desp. 035 Camino del molino, s/n. 28943 Fuenlabrada (Madrid), España antonio.baladron@urjc.es

Beatriz Correyero-Ruiz es doctora en periodismo por la Universidad Complutense de Madrid. Es profesora contratada doctora en la Universidad Católica San Antonio de Murcia (UCAM), donde ejerce como subdirectora del Departamento de Periodismo. Ha impartido las asignaturas de información en radio, periodismo digital y producción periodística. Es además, asesora de la revista Sphera publica y miembro del grupo de investigación en Comunicación y Menores de la UCAM.

Universidad Católica San Antonio Facultad de Comunicación Campus de los Jerónimos, s/n. 30107 Guadalupe (Murcia), España bcorreyero@pdi.ucam.edu

\section{Resumen}

Se presentan los principales resultados de un estudio cualitativo llevado a cabo entre noviembre de 2010 y febrero de 2011 sobre el futuro de las revistas científicas de comunicación en España. La técnica de investigación utilizada fue el método Delphi y se contó con la participación de una muestra significativa de los directores de las principales revistas académicas de comunicación. Las conclusiones revelan las tendencias de futuro más relevantes para estas publicaciones, que en los últimos años han experimentado una gran expansión, tanto en número como en influencia, debido al auge de la investigación comunicológica en España.

\section{Palabras clave}

Revistas científicas, Investigación en comunicación, España, Futuro, Tendencias

\section{Title: Future of communication journals in Spain}

\begin{abstract}
The results of a qualitative investigation carried out between November 2010 and February 2011, on the future of the scientific journals specializing in media and communication in Spain are summarized. The research methodology used was the Delphi method based on the answers provided by the editors of the most important journals in this field. The main purpose was to provide a descriptive analysis of the current situation, along with a prospective analysis to attempt to foresee the future development of these publications.
\end{abstract}

\section{Keywords}

Scientific journals, Media and communication, Research, Communication research, Future, Spain, Trends.

Baladrón-Pazos, Antonio J.; Correyero-Ruiz, Beatriz. "Futuro de las revistas científicas de comunicación en España". El profesional de la información, 2012, enero-febrero, v. 21, n. 1, pp. 34-42. 


\section{Introducción}

El objeto de estudio de este artículo son las revistas científicas de comunicación españolas (en adelante RCCE), que viven una situación de expansión debido al auge de la investigación en comunicación en España. Ese auge comenzó en los años ochenta y fue en los noventa cuando alcanzó su "desarrollo o maduración" (Martínez-Nicolás, 2009). Muestra de la expansión de las RCCE es que entre 2007 y 2008 el número de artículos publicados creció casi un $37 \%$ (Fernández-Quijada, 2010, p. 558) y ese número sigue incrementándose año tras año, ya que en 2007 eran 289 los artículos publicados, en 2008 supusieron un total de 395 y en 2010 fueron ya 495 (Fernández-Quijada, 2011, pp. 3-4). Paralelamente se está produciendo una creciente internacionalización de la investigación en comunicación, incrementándose cada vez más el número de artículos de autores españoles en las revistas de comunicación del Social sciences citation index (SSCl); entre 2005 y 2009 la producción española se incrementó en un 255\% respecto al período 2000-2004 convirtiéndose España en la cuarta nación europea más productiva en ese área (Masip, 2011, pp. 12-13).

La comunicación se suma, aunque con retraso, al boom de la comunicación científica de los años setenta, a menudo sustentando los nuevos proyectos en el libre acceso porque puede ayudar a potenciar el impacto (Abadal; Rius, 2005 , p. 8); cuando menos, incrementa la visibilidad, con la influencia que esto tiene en ocasiones sobre el impacto. Es ahora cuando la relación entre publicaciones e investigación se refuerza con la que Perceval y Fornieles-Alcaraz califican como otra "novedosa" relación (2008, p. 218): la de las publicaciones con las acreditaciones, que actúa sobre la demanda de las RCCE. En este contexto, dado que "la valoración de la calidad exclusivamente a través del impacto es incorrecta" (Aleixandre-Benavent; Valderrama-Zurián; González-Alcaide, 2007, p. 10), se utilizan también otros indicadores alternativos, si bien los índices de impacto están siendo la referencia primaria para los comunicólogos interesados en publicar sus trabajos en las revistas científicas del área y por ende un factor determinante en la demanda de unas cabeceras en detrimento de otras.

Por todo ello, cada vez son más los trabajos sobre este tema, algunos ya citados. Pionero fue el de Giménez-Toledo y Alcaín-Partearroyo (2006), que aportó una primigenia jerarquización de las revistas de periodismo basándose en indicadores de calidad. También de Giménez-Toledo (2011) cabe destacar un reciente estudio basado en una encuesta a expertos en comunicación acerca de la valoración que hacen de las RCCE. Asimismo, hay que referir los estudios bibliométricos de Fernández-Quijada (2010, 2011), que trazan un perfil de las RCCE, Masip (2011), Castillo y Carretón (2010) o Martínez-Nicolás y Saperas-Lapiedra (2011), entre otros. A ellos se suman otros ensayísticos, como los descriptivos de Jones (2003, 2005), fundamentales para un mejor conocimiento de las RCCE. Otros trabajos ensayísticos están más cargados de opinión o son abiertamente críticos (Sabés; Perceval, 2009). Finalmente, existen estudios sobre cabeceras específicas como los de Colle (2009) o López-Ornelas (2010), o bien sobre aspectos particulares de las RCCE, por ejemplo ligados a su naturaleza digital (Martí, 2004; De-
Pablos, 2010). En general, estos trabajos suelen abordar el presente o el pasado de las RCCE y en menor medida el futuro. Igualmente sucede en los estudios sobre el tema realizados en otros países, entre los que no existen precedentes de nuestra investigación en cuanto a los objetivos planteados y la metodología utilizada. Se han publicado trabajos sobre diferentes aspectos de estas revistas científicas como los de Lauf (2005), Feeley (2008) o Espanha y Lima (2011) e incluso en ocasiones se ha utilizado una metodología similar basada en la consulta a expertos (Stumpf, 2008), pero en general están centrados en objetos de estudio más particulares.

La comunicación se suma, con retraso, al boom de la comunicación científica, a menudo sustentando los nuevos proyectos editoriales en políticas de libre acceso

\section{Objetivos y metodología}

El objetivo de nuestra investigación era llevar a cabo de forma científica una prospectiva de las RCCE para los próximos diez años y por ello optamos por el método Delphi, adecuado para prever evoluciones futuras de situaciones cambiantes como la de las publicaciones científicas de comunicación. Es una técnica de previsión subjetiva basada en un proceso iterativo y sistemático en el que un panel de expertos es interrogado sucesivamente con el fin de obtener un consenso de opinión o una opinión grupal fidedigna.

Para seleccionar los expertos se siguieron estos criterios: nivel de conocimientos, grado de motivación, capacidad predictiva y que fuesen especialistas en el tema y estuviesen activamente implicados en él. Se optó por los directivos de las principales RCCE, primer paso que se debía dar para abordar el objeto de estudio, si bien en otras fases actuales del proyecto se está contando con otros grupos de especialistas. Se contactó con las revistas de comunicación presentes en In-Recs (en 2010 eran 21) por ser uno de los principales índices de calidad relativa de las revistas de ciencias sociales. Aunque los índices internacionales tienen mayor relevancia, al estudiarse las cabeceras editadas en España In-Recs era una opción más idónea, además de porque es una referencia inexcusable para las agencias de evaluación y para los investigadores en comunicación. La iniciativa tuvo una excelente acogida; los directores que confirmaron su participación inicial fueron: Ana Azurmendi (Comunicación y sociedad), Felicísimo Valbuena $(\mathrm{ClC})$, Francesc Canosa (Trípodos), Francisco García (/cono14), Humberto MartínezFresneda (Comunicación y hombre), José-Antonio Ortega (Etic@net), J. Ignacio Aguaded (Comunicar), José-Manuel De-Pablos (Latina), Juan Rey (Questiones publicitarias), Luis Núñez (Doxa), Begoña Zabildea (Zer), María-del-Mar Rodríguez (Sphera pública), María-Jesús Casals (Estudios sobre el mensaje periodístico), Miguel Vázquez (I/C) y Ramón Reig (Ámbitos). Concluyeron el proceso, en febrero de 2011, trece expertos y el índice de abandono fue del 13,3\%; en los trabajos publicados se suele situar por encima del $20 \%$ (Lan- 
deta, 2002, p. 122), de modo que no implicó distorsión de los resultados finales.

La principal misión de las revistas de comunicación es ayudar a la acreditación de los investigadores, lo que da cuenta del alcance del llamado efecto Aneca

Se realizaron dos rondas de consultas. En la primera el cuestionario estuvo conformado por preguntas abiertas (tabla 1), dando libertad de respuesta a los expertos. A partir de los datos obtenidos y como corresponde a la ortodoxia del método Delphi se extrajeron los ítems de respuesta del segundo cuestionario, compuesto por preguntas cerradas fruto de la agregación de las opiniones de los expertos y sobre las que se solicitaron valuaciones en una escala $0-5$. No se hizo una tercera ronda por el riesgo de abandono de los expertos y porque científicamente no es necesaria (Landeta, 2002, p. 102). Por último, se sometieron las respuestas recibidas a tratamiento estadístico; al ser valuaciones, se calculó la mediana como medida de tendencia central de los valores de cada ítem de respuesta y éstos se ordenaron de mayor a menor consenso: de mediana superior a inferior y a igual mediana, de menor a mayor desviación típica. De acuerdo con la filosofía del método Delphi, no se ofrecen resultados desagregados por grupos de expertos dado que el propósito era obtener una opinión grupal fidedigna; en todo caso, los niveles de desviación típica en cada ítem dan cuenta del grado de consenso o disenso entre los expertos respecto a cada uno de esos ítems.

\section{Resultados}

En la tabla 2 se recoge la opinión agregada de los expertos sobre la misión que cumplirán las RCCE. Consideran que su papel primordial será ayudar a la acreditación de los investigadores, dato sin duda sorprendente que da cuenta del

\section{Cuestionario Delphi-1}

1. En su opinión ¿cuál es el papel o misión que las RCCE desempeñarán en los próximos diez años en el contexto del Espacio Europeo de Educación Superior? Señale al menos 3 aspectos.

2. Enumere los 5 principales cambios que en los próximos diez años afrontarán las RCCE en su organización interna.

3. ¿Cuáles son los principales servicios que ofrecerán en los próximos diez años las ediciones electrónicas de las RCCE? Indique los 5 que considere más importantes.

4. Señale 5 de las principales medidas que en los próximos diez años adoptarán las RCCE para promover su calidad científica.

5. Enumere 5 de las principales medidas que en los próximos diez años adoptarán las RCCE para promover la consulta y citación de las mismas entre la comunidad científica.

6. Indique cuáles serán los 5 principales criterios para medir el impacto de las RCCE que en los próximos diez años utilizarán las entidades encargadas de esa medición.

7. ¿Cree que en los próximos diez años surgirán más revistas especializadas en comunicación en España? Enumere al menos 3 razones para justificar su opinión.

Tabla 1 alcance del llamado efecto Aneca. Difundir la investigación española en comunicación y promover su internacionalización son otras cuestiones muy valoradas. Con puntuación algo menor los participantes señalan el papel de las RCCE para mejorar la investigación o incrementar el conocimiento en España de las investigaciones comunicológicas que se hacen en otros países. Ser entornos de interacción para investigadores o fuentes de documentación para estudiantes son otras misiones consideradas importantes, si bien los grados de disenso son más altos. En el resto de ítems las desviaciones típicas también son elevadas, evidenciándose la disparidad de opiniones.

Sobre los cambios que afrontarán las RCCE en su organización interna (tabla 3), destaca por su alto consenso la implementación de políticas de acceso libre; todos los expertos apuntan a ello, al igual que dan suma importancia a la sustitución del papel por lo digital -aspecto relacionado-y a la internacionalización de los consejos editoriales. Con similar puntuación, aunque menor consenso, señalan otros aspectos vinculados (reducción de gastos de producción, presencia en redes sociales, mejora de la infraestructura para soportar buenas ediciones electrónicas), así como la necesidad de dedicar más esfuerzos a la inclusión en índices de impacto. En segundo plano, otros cambios son: publicación multilingüe, inclusión de contenidos multimedia, gestión online, universalización de la evaluación por pares o incremento en la información a autores.

Como se preveía que la digitalización era una tendencia en las RCCE, los expertos fueron preguntados por los servicios que ofrecerán las ediciones electrónicas de estas publicaciones. Destacan la búsqueda avanzada en los contenidos y el acceso a los textos completos o la imbricación en la comunicación 2.0. Otras cuestiones sobre las que el consenso

\begin{tabular}{|c|c|c|c|c|}
\hline \multicolumn{5}{|c|}{ Misión en el EEES } \\
\hline Puesto & Ítem & Mediana & Media & Desv. \\
\hline 1 & $\begin{array}{l}\text { Ayudar a la acreditación de los } \\
\text { investigadores }\end{array}$ & 5 & 4,54 & 0,50 \\
\hline 2 & $\begin{array}{l}\text { Difundir la investigación y el } \\
\text { conocimiento en comunicación }\end{array}$ & 5 & 4,46 & 0,63 \\
\hline 3 & $\begin{array}{l}\text { Internacionalizar la investigación } \\
\text { española en comunicación }\end{array}$ & 5 & 4,31 & 0,91 \\
\hline 4 & $\begin{array}{l}\text { Incrementar el conocimiento de } \\
\text { la investigación comunicológica } \\
\text { extranjera }\end{array}$ & 4 & 3,69 & 0,72 \\
\hline 5 & $\begin{array}{l}\text { Contribuir a la innovación y } \\
\text { mejora de la investigación en } \\
\text { comunicación }\end{array}$ & 4 & 3,92 & 0,83 \\
\hline 6 & $\begin{array}{l}\text { Ser entorno de interacción para } \\
\text { investigadores }\end{array}$ & 4 & 4,00 & 1,04 \\
\hline 7 & $\begin{array}{l}\text { Ser fuentes de documentación } \\
\text { para estudiantes universitarios }\end{array}$ & 4 & 3,69 & 1,14 \\
\hline 8 & $\begin{array}{l}\text { Ampliar la cultura editorial cientí- } \\
\text { fica entre los investigadores }\end{array}$ & 4 & 3,77 & 1,37 \\
\hline 9 & $\begin{array}{l}\text { Contribuir a la innovación y mejo- } \\
\text { ra de la docencia universitaria }\end{array}$ & 3 & 3,31 & 1,07 \\
\hline 10 & $\begin{array}{l}\text { Incentivar la competitividad entre } \\
\text { grupos de investigación }\end{array}$ & 3 & 3,54 & 1,15 \\
\hline 11 & $\begin{array}{l}\text { Intensificar las relaciones entre } \\
\text { universidades y empresas }\end{array}$ & 3 & 2,54 & 1,45 \\
\hline 12 & Ser foro de debate sobre el EEES & 3 & 2,46 & 1,55 \\
\hline
\end{tabular}

Tabla 2 
alcanzado es importante son: servicios de acceso a bases de datos, repositorios externos o revistas científicas, versiones en inglés y mayor información sobre sí mismas y su impacto entre los investigadores. En la tabla 4 se detallan otros servicios de interés, como los relativos a la referida gestión y evaluación online.

En cuanto a las medidas que adoptarán las RCCE para promover su calidad científica (tabla 5) sobresale, por la puntuación y el consenso obtenido, lo referente a la colabora-

\begin{tabular}{|c|c|c|c|c|}
\hline \multicolumn{5}{|c|}{ Cambios en organización interna } \\
\hline Puesto & Ítem & Mediana & Media & Desv. \\
\hline 1 & $\begin{array}{l}\text { Acceso abierto y a texto comple- } \\
\text { to en internet }\end{array}$ & 5 & 4,85 & 0,36 \\
\hline 2 & $\begin{array}{l}\text { Ampliación e internacionaliza- } \\
\text { ción de los consejos editoriales }\end{array}$ & 5 & 4,46 & 0,63 \\
\hline 2 & $\begin{array}{l}\text { Progresiva sustitución del papel } \\
\text { por ediciones electrónicas }\end{array}$ & 5 & 4,54 & 0,63 \\
\hline 3 & $\begin{array}{l}\text { Reducción de gastos de produc- } \\
\text { ción }\end{array}$ & 5 & 4,38 & 0,74 \\
\hline 4 & Presencia en redes sociales & 5 & 4,54 & 0,84 \\
\hline 5 & $\begin{array}{l}\text { Mejora de la infraestructura } \\
\text { técnica para soportar buenas } \\
\text { ediciones electrónicas }\end{array}$ & 5 & 4,38 & 0,92 \\
\hline 6 & $\begin{array}{l}\text { Mayor esfuerzo para la inclusión } \\
\text { en índices de impacto }\end{array}$ & 5 & 4,23 & 1,05 \\
\hline 7 & $\begin{array}{l}\text { Publicación en varios idiomas, } \\
\text { fundamentalmente inglés }\end{array}$ & 4 & 4,31 & 0,82 \\
\hline 8 & $\begin{array}{l}\text { Inclusión de contenidos multi- } \\
\text { media }\end{array}$ & 4 & 4,08 & 0,83 \\
\hline 9 & $\begin{array}{l}\text { Realización online de los proce- } \\
\text { sos de gestión y edición }\end{array}$ & 4 & 4,23 & 0,89 \\
\hline 9 & $\begin{array}{l}\text { Universalización del sistema de } \\
\text { evaluación externa, ciega y por } \\
\text { pares }\end{array}$ & 4 & 4,23 & 0,89 \\
\hline 10 & $\begin{array}{l}\text { Más transparencia e información } \\
\text { a los autores en la evaluación }\end{array}$ & 4 & 4,00 & 0,96 \\
\hline 11 & $\begin{array}{l}\text { Mejoras en la promoción y } \\
\text { difusión }\end{array}$ & 4 & 3,85 & 1,03 \\
\hline 11 & $\begin{array}{l}\text { Optimización de los equipos de } \\
\text { evaluadores }\end{array}$ & 4 & 3,85 & 1,03 \\
\hline 12 & $\begin{array}{l}\text { Continuidad o actualización } \\
\text { constante frente a periodicidad } \\
\text { fija }\end{array}$ & 4 & 4,00 & 1,04 \\
\hline 13 & Mejoras en el diseño & 4 & 3,77 & 1,05 \\
\hline 14 & $\begin{array}{l}\text { Mayor exigencia de calidad de los } \\
\text { artículos }\end{array}$ & 4 & 3,69 & 1,14 \\
\hline 15 & $\begin{array}{l}\text { Búsqueda de nuevas fuentes de } \\
\text { financiación }\end{array}$ & 4 & 3,62 & 1,15 \\
\hline 16 & $\begin{array}{l}\text { Mayor profesionalización de los } \\
\text { equipos humanos, principal- } \\
\text { mente en nuevas tecnologías o } \\
\text { índices de impacto }\end{array}$ & 4 & 3,69 & 1,20 \\
\hline 17 & $\begin{array}{l}\text { Mayor garantía del anonimato en } \\
\text { las evaluaciones }\end{array}$ & 4 & 3,46 & 1,39 \\
\hline 18 & $\begin{array}{l}\text { Consolidación de acuerdos entre } \\
\text { revistas }\end{array}$ & 4 & 3,69 & 1,49 \\
\hline 19 & $\begin{array}{l}\text { Renuncia al anonimato de los } \\
\text { evaluadores }\end{array}$ & 3 & 3,38 & 1,27 \\
\hline 20 & $\begin{array}{l}\text { Mayor preocupación por los } \\
\text { derechos de autor }\end{array}$ & 3 & 2,92 & 1,33 \\
\hline
\end{tabular}

Tabla 3 ción de las revistas en los sistemas de medición del impacto mediante proyectos conjuntos y al mayor cumplimiento de los requisitos para su indexación. Si lo segundo tiene más que ver con la implementación por parte de cada revista de las medidas para cumplir con los requisitos que los sistemas de medición del impacto exigen, lo primero hace referencia al establecimiento de cauces de colaboración entre las distintas cabeceras encaminados a coordinar colectivamente sus políticas de gestión editorial armonizándolas con las contempladas por esos sistemas de medición del impacto; de hecho, ya existen iniciativas conjuntas de las que forman parte las principales revistas y con las que se pretende una mayor fuerza colectiva a la hora de conseguir un mayor peso de las RCCE.

Por otra parte, el impulso a las ediciones electrónicas y al acceso abierto son, una vez más, otras dos medidas fundamentales para los expertos, así como el cumplimento escrupuloso de los procedimientos de evaluación. Muy importantes, aunque con menor consenso, son: la utilización de criterios más objetivos y claros por parte de las propias revistas en los procesos de evaluación de los artículos, la potenciación de investigación innovadora y aplicada, la lucha contra lo que consideran prácticas corruptas de algunas cabeceras para favorecer sus índices de impacto, prácticas que algunos de los expertos participantes ejemplifican con

\begin{tabular}{|c|c|c|c|c|}
\hline \multicolumn{5}{|c|}{ Servicios de ediciones electrónicas } \\
\hline Puesto & Ítem & Mediana & Media & Desv. \\
\hline 1 & $\begin{array}{l}\text { Búsqueda avanzada en los } \\
\text { contenidos }\end{array}$ & 5 & 4,31 & 0,91 \\
\hline 2 & Acceso a texto completo & 5 & 4,46 & 1,08 \\
\hline 3 & Acceso a redes sociales & 5 & 4,23 & 1,19 \\
\hline 4 & $\begin{array}{l}\text { Acceso a bases de datos o repo- } \\
\text { sitorios externos }\end{array}$ & 4 & 4,23 & 0,70 \\
\hline 5 & $\begin{array}{l}\text { Acceso a otras revistas cientí- } \\
\text { ficas }\end{array}$ & 4 & 4,23 & 0,80 \\
\hline 6 & $\begin{array}{l}\text { Versiones en varios idiomas, } \\
\text { fundamentalmente inglés }\end{array}$ & 4 & 4,23 & 0,89 \\
\hline 7 & $\begin{array}{l}\text { Exhaustiva información sobre la } \\
\text { revista (impacto, normas, etc.) }\end{array}$ & 4 & 4,15 & 0,95 \\
\hline 8 & $\begin{array}{l}\text { Consulta en línea del estado } \\
\text { de los originales sometidos a } \\
\text { evaluación }\end{array}$ & 4 & 3,94 & 1,00 \\
\hline 9 & $\begin{array}{l}\text { Foros para la interacción entre } \\
\text { investigadores }\end{array}$ & 4 & 3,77 & 1,05 \\
\hline 10 & $\begin{array}{l}\text { Envío y autoedición online de } \\
\text { los artículos }\end{array}$ & 4 & 4,08 & 1,07 \\
\hline 11 & $\begin{array}{l}\text { Incorporación de nuevos forma- } \\
\text { tos en los artículos }\end{array}$ & 4 & 3,77 & 1,12 \\
\hline 12 & $\begin{array}{l}\text { Mayor interacción con directi- } \\
\text { vos o evaluadores }\end{array}$ & 4 & 3,85 & 1,23 \\
\hline 13 & $\begin{array}{l}\text { Informaciones útiles para inves- } \\
\text { tigadores (congresos, call for } \\
\text { papers, links de interés, etc.) }\end{array}$ & 4 & 3,77 & 1,25 \\
\hline 13 & $\begin{array}{l}\text { Continuidad y actualización } \\
\text { constante frente a periodicidad } \\
\text { fija }\end{array}$ & 4 & 3,77 & 1,25 \\
\hline 14 & $\begin{array}{l}\text { Información personalizada a } \\
\text { cada lector }\end{array}$ & 4 & 3,46 & 1,39 \\
\hline 15 & Más números monográficos & 4 & 3,23 & 1,53 \\
\hline 16 & Más secciones temáticas & 4 & 3,23 & 1,58 \\
\hline
\end{tabular}

Tabla 4 


\begin{tabular}{|c|c|c|c|c|}
\hline \multicolumn{5}{|c|}{ Medidas para promover la calidad científica } \\
\hline Puesto & Ítem & Mediana & Media & Desv. \\
\hline 1 & $\begin{array}{l}\text { Impulsar las ediciones electró- } \\
\text { nicas }\end{array}$ & 5 & 4,62 & 0,62 \\
\hline 2 & $\begin{array}{l}\text { Cumplir con puntualidad escru- } \\
\text { pulosa los procedimientos }\end{array}$ & 5 & 4,46 & 0,63 \\
\hline 2 & $\begin{array}{l}\text { Cumplir los requisitos para estar } \\
\text { indexadas en índices de refe- } \\
\text { rencia }\end{array}$ & 5 & 4,54 & 0,63 \\
\hline 3 & $\begin{array}{l}\text { Colaborar en los sistemas de } \\
\text { medición del impacto }\end{array}$ & 5 & 4,38 & 0,74 \\
\hline 4 & $\begin{array}{l}\text { Mejorar la accesibilidad a los } \\
\text { contenidos en internet }\end{array}$ & 5 & 4,31 & 0,91 \\
\hline 5 & $\begin{array}{l}\text { Utilizar criterios de evaluación } \\
\text { de artículos más objetivos, } \\
\text { claros y públicos }\end{array}$ & 5 & 3,92 & 1,33 \\
\hline 6 & $\begin{array}{l}\text { Potenciar la investigación inno- } \\
\text { vadora aplicada y de interés } \\
\text { social }\end{array}$ & 5 & 3,92 & 1,49 \\
\hline 7 & $\begin{array}{l}\text { Luchar contra prácticas corrup- } \\
\text { tas de las propias cabeceras } \\
\text { para entrar y mantenerse en } \\
\text { índices de impacto }\end{array}$ & 5 & 4,00 & 1,62 \\
\hline 8 & $\begin{array}{l}\text { Fomentar los servicios de } \\
\text { búsqueda avanzada en los } \\
\text { contenidos }\end{array}$ & 4 & 4,15 & 0,86 \\
\hline 9 & $\begin{array}{l}\text { Abrir espacios de debate para } \\
\text { los investigadores }\end{array}$ & 4 & 4,08 & 0,92 \\
\hline 9 & $\begin{array}{l}\text { Afrontar la profesionalización } \\
\text { de los procesos de producción } \\
\text { (redacción, diseño) dejando los } \\
\text { contenidos a los comunicólogos }\end{array}$ & 4 & 4,08 & 0,92 \\
\hline 10 & $\begin{array}{l}\text { Renovar la infraestructura } \\
\text { técnica para soportar buenas } \\
\text { ediciones electrónicas }\end{array}$ & 4 & 4,15 & 0,95 \\
\hline 11 & Editar en inglés & 4 & 3,69 & 0,99 \\
\hline 12 & $\begin{array}{l}\text { Incrementar el número de } \\
\text { revistas }\end{array}$ & 4 & 3,69 & 1,07 \\
\hline 12 & $\begin{array}{l}\text { Internacionalizar los call for } \\
\text { papers y los colaboradores }\end{array}$ & 4 & 3,69 & 1,07 \\
\hline 12 & $\begin{array}{l}\text { Unificar las normas editoriales y } \\
\text { de evaluación entre las distintas } \\
\text { revistas }\end{array}$ & 4 & 3,69 & 1,07 \\
\hline 13 & $\begin{array}{l}\text { Exigir el cumplimiento absoluto } \\
\text { de requisitos formales en las } \\
\text { colaboraciones }\end{array}$ & 4 & 3,85 & 1,17 \\
\hline 13 & $\begin{array}{l}\text { Incluir documentación audiovi- } \\
\text { sual en los artículos }\end{array}$ & 4 & 3,85 & 1,17 \\
\hline 14 & $\begin{array}{l}\text { Mejorar la transparencia e inte- } \\
\text { ractividad con los autores }\end{array}$ & 4 & 4,00 & 1,18 \\
\hline 15 & $\begin{array}{l}\text { Exigir que los artículos se basen } \\
\text { en metodología científica }\end{array}$ & 4 & 4,00 & 1,24 \\
\hline 15 & Mejorar el diseño y edición & 4 & 4,00 & 1,24 \\
\hline 16 & $\begin{array}{l}\text { Incrementar el nivel académico } \\
\text { de los consejos editoriales }\end{array}$ & 4 & 3,69 & 1,26 \\
\hline 17 & $\begin{array}{l}\text { Dar mayor importancia a las } \\
\text { reseñas de libros de calidad }\end{array}$ & 4 & 3,69 & 1,32 \\
\hline 18 & Utilizar el sistema DOI & 4 & 3,85 & 1,35 \\
\hline 19 & $\begin{array}{l}\text { Valorar la actualización e } \\
\text { internacionalización de las } \\
\text { bibliografías }\end{array}$ & 4 & 3,77 & 1,42 \\
\hline 20 & $\begin{array}{l}\text { Extremar las exigencias en la } \\
\text { evaluación }\end{array}$ & 4 & 3,85 & 1,46 \\
\hline 20 & $\begin{array}{l}\text { Universalizar la evaluación } \\
\text { externa, ciega y por pares }\end{array}$ & 4 & 3,85 & 1,46 \\
\hline
\end{tabular}

la promoción excesiva de la autocitación. Este último aspecto evidencia los recelos de algunos directivos consultados respecto al comportamiento de otras revistas distintas a la suya.

Otros aspectos ya señalados también son considerados relevantes para promover la calidad: servicios de búsqueda avanzada, espacios para la interacción entre los investigadores, profesionalización de los procesos productivos, mejora de las ediciones electrónicas o edición en inglés.

\begin{tabular}{|c|c|c|c|c|}
\hline \multicolumn{5}{|c|}{ Medidas para promover citación } \\
\hline Puesto & Ítem & Mediana & Media & Desv. \\
\hline 1 & Optimizar la edición electrónica & 5 & 4,62 & 0,62 \\
\hline 2 & $\begin{array}{l}\text { Colaborar en los sistemas de } \\
\text { medición del impacto }\end{array}$ & 5 & 4,38 & 0,84 \\
\hline 3 & $\begin{array}{l}\text { Mejorar el posicionamiento en } \\
\text { buscadores }\end{array}$ & 5 & 4,31 & 0,91 \\
\hline 4 & Intensificar la promoción & 5 & 4,54 & 0,93 \\
\hline 4 & $\begin{array}{l}\text { Incrementar el uso de comuni- } \\
\text { cación } 2.0\end{array}$ & 5 & 4,54 & 0,93 \\
\hline 5 & $\begin{array}{l}\text { Luchar contra prácticas corrup- } \\
\text { tas de las propias cabeceras } \\
\text { para entrar y mantenerse en } \\
\text { índices de impacto }\end{array}$ & 5 & 4,46 & 1,39 \\
\hline 6 & $\begin{array}{l}\text { Incorporarse a sistemas de } \\
\text { indexación internacionales } \\
\text { solventes }\end{array}$ & 5 & 4,15 & 1,51 \\
\hline 7 & $\begin{array}{l}\text { Editar en varios idiomas, funda- } \\
\text { mentalmente inglés }\end{array}$ & 4 & 4,08 & 0,83 \\
\hline 8 & $\begin{array}{l}\text { Enviar avisos de citas localizadas } \\
\text { a los autores }\end{array}$ & 4 & 3,92 & 1,07 \\
\hline 8 & $\begin{array}{l}\text { Incorporarse a directorios del } \\
\text { tipo DOAJ }\end{array}$ & 4 & 4,08 & 1,07 \\
\hline 9 & $\begin{array}{l}\text { Enviar información personaliza- } \\
\text { da a los investigadores }\end{array}$ & 4 & 3,54 & 1,15 \\
\hline 10 & $\begin{array}{l}\text { Organizar y participar en en- } \\
\text { cuentros sobre comunicación }\end{array}$ & 4 & 3,69 & 1,2 \\
\hline 11 & $\begin{array}{l}\text { Garantizar el rigor y la exigencia } \\
\text { en la selección de artículos }\end{array}$ & 4 & 4,08 & 1,21 \\
\hline 11 & $\begin{array}{l}\text { Incorporar profesionales espe- } \\
\text { cializados en índices de calidad }\end{array}$ & 4 & 3,92 & 1,21 \\
\hline 12 & $\begin{array}{l}\text { Fomentar la innovación en la } \\
\text { investigación en comunicación }\end{array}$ & 4 & 4,00 & 1,24 \\
\hline 13 & $\begin{array}{l}\text { Utilizar prescriptores o reco- } \\
\text { mendadores de contenido }\end{array}$ & 4 & 3,69 & 1,32 \\
\hline 14 & $\begin{array}{l}\text { Disponer de metadatos del tipo } \\
\text { Dublin Core y exponerlos a los } \\
\text { lectores }\end{array}$ & 4 & 3,62 & 1,33 \\
\hline 15 & $\begin{array}{l}\text { Mejorar el nivel investigador de } \\
\text { los autores }\end{array}$ & 4 & 3,77 & 1,37 \\
\hline 16 & $\begin{array}{l}\text { Promover asociaciones entre } \\
\text { cabeceras }\end{array}$ & 4 & 3,69 & 1,43 \\
\hline 17 & $\begin{array}{l}\text { Incluir recomendaciones a } \\
\text { artículos relacionados }\end{array}$ & 4 & 3,38 & 1,44 \\
\hline 18 & Aumentar la periodicidad & 4 & 3,31 & 1,64 \\
\hline 19 & $\begin{array}{l}\text { Promover la citación de la revis- } \\
\text { ta entre los colaboradores }\end{array}$ & 4 & 2,92 & 1,73 \\
\hline 20 & $\begin{array}{l}\text { Intensificar la distribución física } \\
\text { y los intercambios }\end{array}$ & 3 & 2,77 & 2,04 \\
\hline
\end{tabular}

Tabla 6 
Dada la relación entre calidad e impacto, cuando los expertos fueron interrogados sobre medidas que adoptarán para promover la citación, parte de las respuestas más valoradas siguen la misma línea que las recogidas en la tabla 5: mejorar las ediciones electrónicas, colaborar en los sistemas de medición del impacto evitando prácticas corruptas por parte de las propias cabeceras, incorporarse a bases de datos internacionales, editar en inglés, etc. Pero también valoran otras como mejorar el posicionamiento web, dedicar más esfuerzos a la promoción o incorporarse a plataformas de comunicación 2.0; en definitiva, potenciar las políticas de marketing. Otras medidas a las que los participantes en el Delphi conceden cierta relevancia son las relativas al envío de información a los investigadores sobre los artículos publicados y las citas localizadas.

Respecto a los criterios que utilizarán las entidades encargadas de la medición del impacto de las RCCE llama la atención que la respuesta mejor valorada sea la referida a los índices de lectura o difusión de las revistas, aunque el elevado disenso permite relativizar este dato. Otros de los criterios apuntados tienen más que ver con los referentes

\begin{tabular}{|c|l|c|c|c|}
\hline \multicolumn{5}{|c}{ Criterios para medir impacto } \\
\hline Puesto & \multicolumn{1}{|c|}{ Ítem } & Mediana & Media & Desv. \\
\hline 1 & $\begin{array}{l}\text { Índices de lectura o difusión: con- } \\
\text { sultas, descargas online, etc. }\end{array}$ & 5 & 4,23 & 1,31 \\
\hline 2 & $\begin{array}{l}\text { Variedad de las citas a la revista } \\
\text { (en otras áreas, etc.) }\end{array}$ & 4 & 4,08 & 0,83 \\
\hline 3 & $\begin{array}{l}\text { Nivel de transparencia en los } \\
\text { procesos de evaluación }\end{array}$ & 4 & 4,15 & 0,86 \\
\hline 4 & $\begin{array}{l}\text { Existencia de evaluadores exter- } \\
\text { nos, ciegos y por pares }\end{array}$ & 4 & 4,23 & 0,89 \\
\hline 5 & $\begin{array}{l}\text { Nivel investigador del consejo } \\
\text { editorial }\end{array}$ & 4 & 4,08 & 0,92 \\
\hline 5 & Número de citas en otras revistas & 4 & 3,92 & 0,92 \\
\hline 6 & $\begin{array}{l}\text { Presencia en la web 2.0: blogs, } \\
\text { redes sociales }\end{array}$ & 4 & 4,15 & 0,95 \\
\hline 6 & $\begin{array}{l}\text { Rigor y cumplimiento de criterios } \\
\text { de calidad regulados }\end{array}$ & 4 & 4,15 & 0,95 \\
\hline 7 & $\begin{array}{l}\text { Edición en varios idiomas, funda- } \\
\text { mentalmente inglés }\end{array}$ & 4 & 3,77 & 0,97 \\
\hline 8 & $\begin{array}{l}\text { Credibilidad e influencia entre la } \\
\text { comunidad científica }\end{array}$ & 4 & 4,08 & 1,00 \\
\hline 8 & Regularidad en la publicación & 4 & 4,08 & 1,00 \\
\hline 9 & $\begin{array}{l}\text { Número de citas en actas de } \\
\text { congresos de excelencia }\end{array}$ & 4 & 3,85 & 1,03 \\
\hline 9 & $\begin{array}{l}\text { Número de citas en publicacio- } \\
\text { nes de otros países o idiomas }\end{array}$ & 4 & 3,85 & 1,03 \\
\hline 10 & Apertura editorial & 4 & 4,00 & 1,04 \\
\hline 11 & $\begin{array}{l}\text { Presencia en actos académicos: } \\
\text { congresos, etc. }\end{array}$ & 4 & 3,69 & 1,14 \\
\hline 12 & Nivel investigador de los autores & 4 & 4,08 & 1,21 \\
\hline 13 & $\begin{array}{l}\text { Fuentes mencionadas por los } \\
\text { autores }\end{array}$ & 4 & 3,77 & 1,31 \\
\hline 14 & $\begin{array}{l}\text { Impacto social de las investiga- } \\
\text { ciones publicadas }\end{array}$ & 4 & 3,85 & 1,35 \\
\hline 14 & $\begin{array}{l}\text { Originalidad de las contribucio- } \\
\text { nes }\end{array}$ & 4 & 3,85 & 1,35 \\
\hline & Número de artículos publicados & 3 & 3,31 & 1,20 \\
\hline
\end{tabular}

Tabla 7 actuales: número y diversidad de citas, transparencia en las evaluaciones y evaluación por pares, nivel académico de los consejos editoriales, cumplimiento de criterios de calidad regulados, así como edición en inglés.

Finalmente se interrogó a los expertos sobre el nacimiento de nuevas cabeceras en los próximos diez años y la respuesta afirmativa fue unánime. Según indican, sucederá sobre todo para dar respuesta a la necesidad de publicar que tiene la creciente comunidad de comunicólogos. También afirman que influirán los bajos costes de las ediciones electrónicas o su utilidad para dar visibilidad a grupos de investigación emergentes. En la tabla 8 se recogen otras razones, muy vinculadas al auge de la investigación en comunicación que se vive en España.

Entre los cambios que afrontarán en los próximos diez años las RCCE, destacan la implementación de políticas de libre acceso o la sustitución del papel por lo digital

\section{Conclusiones}

De las opiniones de los expertos consultados se desprende cierta falta de madurez y profesionalización de las RCCE. De hecho, algunas de las tendencias de futuro señaladas son práctica habitual en las revistas científicas de otras áreas. Es el caso de la universalización del sistema de evaluación ciega por pares y de una mayor internacionalización de los consejos editoriales, además de ser necesarios en general mayores esfuerzos para la inclusión en índices de impacto. Puede explicarse por la propia juventud e inmadurez de la disciplina, caracterizada por una "insuficiencia metodológica generalizada" (Martínez-Nicolás y Saperas-Lapiedra, 2011, p. 124), y por la urgencia que los procesos de evaluación de

\begin{tabular}{|c|c|c|c|c|}
\hline \multicolumn{5}{|c|}{ Razones para nuevas cabeceras } \\
\hline Puesto & Ítem & Mediana & Media & Desv. \\
\hline 1 & $\begin{array}{l}\text { Para dar respuesta a la necesidad } \\
\text { de publicar de los investigadores } \\
\text { pendientes de acreditación }\end{array}$ & 5 & 4,46 & 0,75 \\
\hline 2 & $\begin{array}{l}\text { Por la disminución de costes de la } \\
\text { edición electrónica }\end{array}$ & 5 & 4,15 & 1,17 \\
\hline 3 & $\begin{array}{l}\text { Porque son útiles para dar visibili- } \\
\text { dad a grupos de investigación }\end{array}$ & 5 & 4,23 & 1,25 \\
\hline 4 & $\begin{array}{l}\text { Por el incremento de centros e } \\
\text { investigadores especializados en } \\
\text { comunicación }\end{array}$ & 4 & 3,85 & 1,03 \\
\hline 5 & $\begin{array}{l}\text { Porque hay cabida para revistas } \\
\text { especializadas en áreas de la co- } \\
\text { municación poco representadas }\end{array}$ & 4 & 3,92 & 1,27 \\
\hline 6 & $\begin{array}{l}\text { Por la mayor valoración de la } \\
\text { investigación comunicológica } \\
\text { entre los estamentos científicos }\end{array}$ & 4 & 3,77 & 1,48 \\
\hline 7 & $\begin{array}{l}\text { Por la importancia de la comuni- } \\
\text { cación en la sociedad }\end{array}$ & 3 & 3,62 & 1,27 \\
\hline 8 & $\begin{array}{l}\text { Por el incremento en el número } \\
\text { de lectores }\end{array}$ & 3 & 3,08 & 1,38 \\
\hline
\end{tabular}

Tabla 8 
la actividad investigadora imprimen a la evolución reciente de estas publicaciones.

Algunas cabeceras se han encontrado con un importante desconocimiento respecto al conjunto de medidas y dinámicas que adoptar para mejorar su impacto, por lo que sería recomendable un mayor asesoramiento de documentalistas y profesionales de la información. Si bien es incuestionable que la gestión de los editores para mantener niveles exigentes de calidad científica de los contenidos de sus revistas es la principal vía que puede servir para consolidar un mayor impacto de las mismas, lo cierto es que en los últimos años, por ese insuficiente conocimiento sobre el tema, muchas RCCE han tardado en incorporar a su gestión las necesarias medidas y requisitos para tener más posibilidades de un mayor impacto. Es una situación que, de hecho, se ha empezado a corregir en algunos casos en los que los editores han buscado la asesoría de expertos en el campo, por supuesto paralelamente a una adecuada gestión en el nivel de calidad científica de los contenidos.

El futuro pasará por optimizar los procesos de evaluación de los artículos, ya que las respuestas de los expertos evidencian que los procedimientos no son siempre suficientemente rigurosos. No en vano una de las medidas que proponen para promover la calidad científica es la utilización de criterios de evaluación de los artículos más objetivos, públicos y claros por parte de las propias revistas; si bien es cierto que esto es una realidad cada vez más constatable, dicha opinión podría estar relacionada con esas carencias que, según indican, persisten en algunas publicaciones en lo referente a la evaluación anónima y transparente ante los autores.

Hay cierta falta de madurez de las RCCE, ya que algunas de las tendencias de futuro señaladas ya son práctica habitual en las revistas científicas de otras áreas

También urge ampliar las posibilidades que ofrecen las ediciones electrónicas. Salvo en casos excepcionales, los expertos consideran que los servicios que ofrecen (búsqueda avanzada, información sobre la propia revista, gestión online, etc.) son muy deficientes, cuando no inexistentes, quedando al descubierto el retraso tecnológico que sufren debido a los problemas de financiación o a la falta de personal especializado en nuevas tecnologías. El futuro implicará un mayor impulso a la digitalización favorecida por el consiguiente ahorro en los costes de edición, factor importante para muchas cabeceras. Además, según los especialistas, esa digitalización irá imbricada en políticas de libre acceso. La juventud de muchas RCCE y las características propias de este mercado editorial justifican que a corto plazo el acceso abierto se imponga para permitir una mayor visibilidad e intentar con ello potenciar un mayor impacto de las revistas. A diferencia de lo que ocurre en otros mercados en los que es práctica más habitual el acceso restringido y un mayor sometimiento a los intereses lucrativos de las empresas editoras, casi todas las RCCE son editadas por instituciones públicas.
Por otra parte, cada vez será más necesaria la edición en inglés; así se desprende de la opinión de los encuestados en varias preguntas del cuestionario, aunque hay que aclarar que a lo que la práctica totalidad de ellos se refiere es a la edición bilingüe en inglés y español, precisamente para abarcar una comunidad científica más amplia sin menoscabo de la relevancia que cualquiera de estos dos idiomas pueda tener como vehículo de comunicación científica en distintas áreas geográficas. Cabe señalar, en todo caso, una cierta confusión respecto a la relación entre la calidad científica de las revistas y la edición en inglés. De la lectura literal de los datos obtenidos en la investigación se podría concluir que vinculan estos aspectos como si el idioma utilizado condicionase la calidad, cuando de una lectura más global de los cuestionarios se podría deducir que relacionan la utilización del inglés con la mayor visibilidad de las cabeceras y por tanto con mayores posibilidades para acreditar su impacto o calidad; de hecho, la edición en inglés también es subrayada cuando los expertos son preguntados sobre las medidas que adoptarán las RCCE para incrementar su impacto. Además, al referirse a las medidas para promover la calidad científica también se señalan como destacados otros factores vinculados a la visibilidad antes que a la calidad en sí misma, lo cual se podría explicar por esa equiparación que hacen entre visibilidad y calidad científica, aunque en el cuestionario no se exprese como tal. Prueba de ello es que hay especialistas que aportan algunas respuestas coincidentes en las dos preguntas, lo que ha contribuido a esta distorsión, mientras que otros han separado claramente ambos aspectos, por lo que podría ser necesario en próximas fases del estudio implementar medidas para evitar esa distorsión. En todo caso, se exponen objetivamente esos datos porque se han querido redactar las opiniones agregadas recogidas en el segundo cuestionario del Delphi manteniendo el sentido de las respuestas dadas al primero y sin perder información real, para cumplir así con las exigencias de esta técnica de investigación.

\section{El futuro de las revistas científicas de co- municación pasa por una mayor interna- cionalización y mayores esfuerzos para la inclusión en índices de impacto}

En la investigación realizada se dejan entrever algunas críticas hacia el comportamiento de las RCCE con relación a los sistemas de evaluación bibliométrica de la calidad científica de las revistas. Así, en varias ocasiones se habla literalmente de la necesidad de superar prácticas corruptas de las propias cabeceras para favorecer sus índices de impacto, refiriéndose a la autocitación y promoción de las citas entre colaboradores. Aunque en las tablas se recogió la opinión agregada, analizando las opiniones individuales se comprueba la disparidad de pareceres al respecto, como queda patente en las desviaciones típicas referidas a estos ítems de respuesta. Existe un grupo minoritario de expertos que critica esos sistemas de evaluación bibliométrica de las revistas, pero sobre todo en lo referente a las posiciones adoptadas al respecto por algunas cabeceras, mientras que otros defienden esas posiciones como válidas. En muchas ocasiones 
estas críticas hacia el propio sector editorial son achacadas a razones externas, principalmente a imposiciones de las entidades encargadas de esa evaluación, si bien los propios encuestados son también autocríticos con muchas carencias que arrastran las RCCE, algunas de las cuales se han indicado anteriormente. En cualquier caso y en contraste con algunas de esas opiniones de los participantes en el Delphi, conviene subrayar el importante papel que, a pesar de las limitaciones que puedan tener, cumplen los sistemas de valoración de la investigación científica a partir de indicadores bibliométricos para garantizar un proceso lo menos arbitrario posible en la evaluación y acreditación de la producción científica de los investigadores y para contribuir a una mayor excelencia de la comunidad científica.

Pese a las carencias señaladas en párrafos anteriores, las RCCE tendrán un papel cada vez más importante en la difusión de la investigación en comunicación si bien para los expertos consultados su principal utilidad práctica será la de ayudar a los procesos de acreditación. El objetivo de esta investigación era recoger la opinión agregada de los directores de las principales revistas y de ahí lo significativo de este dato, ya que sin duda resulta sorprendente que consideren que sus cabeceras son herramienta para la acreditación de los investigadores antes que para promover la difusión del conocimiento científico; sobre todo por lo que ello implica en la definición de los objetivos, estrategias y tácticas que marcarán en los próximos años para el desarrollo de esas cabeceras y la misión que le asignarán a las mismas. El estudio Delphi ha permitido constatar esa opinión, pero sin duda sería necesario profundizar en éste y otros datos con una investigación cualitativa complementaria.

También es llamativo que los expertos defiendan la creación de nuevas RCCE para dar visibilidad a grupos de investigación, cuando, lejos de procedimientos endogámicos, las revistas científicas debieran ser vehículos abiertos y competitivos, nunca cerrados a los intereses de equipos de investigación más o menos emergentes.

Por último, cabe destacar la unanimidad de los expertos respecto a la necesidad de que surjan nuevas cabeceras en los próximos diez años. La principal razón es el crecimiento exponencial de la comunidad científica de investigadores en comunicación con la implantación de estos estudios en medio centenar de centros universitarios. Ahora bien, mientras la mayoría de esos investigadores pugnan por publicar en las principales revistas del área, otras revistas tienen problemas para recibir originales de calidad. Por ello, hay que valorar la tendencia contraria. La reconfiguración del mercado de RCCE tendrá que ir paralela a un incremento aún mayor en la calidad global de las publicaciones, siendo necesario, como se concluye en un reciente estudio (López-Rabadán; Vicente-Mariño, 2011, p. 13), "una fase de apuntalamiento, en la que las revistas con mayor bagaje deben ejercer una función de referencia". En la misma línea, Giménez-Toledo (2011, p. 8) aboga por una "agrupación de revistas generalistas" que permitiría concentrar esfuerzos. La mayor madurez del conjunto de las RCCE contribuiría sin duda a la consolidación de algunas de las publicaciones emergentes surgidas en los últimos años, así como al prestigio de otras cabeceras más veteranas que empiezan ya a ser conscientes de la importancia de que su calidad sea acreditada mediante procedimientos de evaluación bibliométrica.

\section{Bibliografía}

Abadal, Ernest; Rius, Lluís. "Revistas científicas digitales: características e indicadores". Revista de universidad y sociedad del conocimiento, 2006, v. 3, n. 1, pp. 6-20. http://www.uoc.edu/rusc/3/1/dt/esp/abadal_rius.pdf

Aleixandre-Benavent, Rafael; Valderrama-Zurián, JuanCarlos; González-Alcaide, Gregorio. "El factor de impacto de las revistas científicas: limitaciones e indicadores alternativos". El profesional de la información, 2007, v. 16, n. 1, pp. 4-11.

http://eprints.rclis.org/handle/10760/9489

Castillo, Antonio; Carretón, María-del-Carmen. "Investigación en comunicación. Estudio bibliométrico de las revistas de comunicación en España". Comunicación y sociedad, 2010, v. 23, n. 2, pp. 289-327.

http://www.unav.es/fcom/comunicacionysociedad/es/ articulo.php?art_id=372

Colle, Raymond. "La temática de Revista latina de comunicación social, 1998-2008". Revista latina de comunicación social, 2009, n. 64, pp. 71-85.

http://www.ull.es/publicaciones/latina/09/art/07_806_ 13_revista/Raymond_Colle.html

De-Pablos, José-Manuel. "Análisis de las revistas españolas de comunicación tras la actualización del índice de impacto de 2009". En: // Congreso intl Latina de comunicación social, 2010. ISBN: 9788493842802

http://www.revistalatinacs.org/10SLCS/actas_2010/217_ De_Pablos.html

Espanha, Rita; Lima, Tiago. “Open access and multilingual approach to communication journals. The case and the editor's perspective of Observatorio journal and the importance of open science for the knowledge society". Online journal of communication and media technologies, 2011, v. 1, n. 4, pp. 97-120.

http://ojcmt.net/articles/144.pdf

Feeley, Thomas-Hugh. "A bibliometric analysis of communication journals from 2002 to 2005". Human communication research, 2008, v. 34, n. 3, pp. 505-520.

http://www.iimahd.ernet.in/library/PDFs/NICMAN/A bibleo metric analysis of communication journals.pdf

Fernández-Quijada, David. "El perfil de las revistas españolas de comunicación (2007-2008)". Revista española de documentación científica, 2010, v. 33, n. 4, pp. 553-581.

http://redc.revistas.csic.es/index.php/redc/article/down load/671/746

http://dx.doi.org/10.3989/redc.2010.4.756

Fernández-Quijada, David. “De los investigadores a las redes. Una aproximación tipológica a la autoría en las revistas españolas de comunicación". En: Simposio AE-IC Investigar la comunicación en España, 2011.

http://eprints.rclis.org/handle/10760/15566

Giménez-Toledo, Elea; Alcaín-Partearroyo, Dolores. “Estu- 
dio de las revistas españolas de periodismo". Comunicación y sociedad, 2006, v. 19, n. 2, pp. 107-131.

http://www.unav.es/fcom/comunicacionysociedad/es/ articulo.php?art_id $=62$

Giménez-Toledo, Elea. "La opinión de los expertos sobre las revistas españolas de comunicación y otros indicadores de calidad". En: Simposio AE-IC Investigar la comunicación en España, 2011.

http://www.revistacomunicar.com/pdf/2011-04-EleaGimenez.pdf

Jones, Daniel. "Les revistes teòriques sobre comunicació audiovisual a Iberoamèrica". Quaderns del CAC, 2003, n. 17, pp. 57-66.

http://www.cac.cat/pfw_files/cma/recerca/quaderns_cac/ Q17jones.pdf

Jones, Daniel. "Las revistas de comunicación en España". Telos, 2005, n. 64.

http://www.sociedadinformacion.fundacion.telefonica. com/telos/articulorevista.asp@idarticulo=1\&rev=64.htm

Landeta, Jon. El método Delphi. Barcelona: Ariel, 1999. ISBN 8434428369

Lauf, Edmund. "National diversity of major international journals in the field of communication". Journal of communication, 2005, v. 55, n. 1, pp. 139-151.

http://newmedia.cityu.edu.hk/enjhzhu/ruc/readings/Lauf_ 2005_NationalDiversity.pdf

López-Ornelas, Maricela. "Estudio cuantitativo de los procesos de comunicación de Revista latina de comunicación social, 1998-2009". Revista latina de comunicación social, 2010, n. 65, pp. 538-552.

http://www.revistalatinacs.org/10/art3/917_Mexico/39_ Maricela.html

http://dx.doi.org/10.4185/RLCS-65-2010-917-538-552

López-Rabadán, Pablo; Vicente-Mariño, Miguel. "Métodos y técnicas de investigación dominantes en las revistas científicas españolas sobre comunicación (2000-2009)". En: Simposio AE-IC Investigar la comunicación en España, 2011. http://www.revistacomunicar.com/pdf/2011-04-LopezVicente.pdf
Martí, Daniel. "Las revistas académicas ibéricas y latinoamericanas de comunicación en internet en el contexto tecnológico actual". Razón y palabra, 2004, n. 41.

http://www.razonypalabra.org.mx/anteriores/n41/dmarti. html

Martínez-Nicolás, Manuel. "La investigación sobre comunicación en España. Evolución histórica y retos actuales". Revista latina de comunicación social, 2009, n. 64, pp. 1-14. http://www.ull.es/publicaciones/latina/09/art/01_800_ 01_investigacion/Manuel_Martinez_Nicolas.html

Martínez-Nicolás, Manuel; Saperas-Lapiedra Enric. "La investigación sobre comunicación en España (1998-2007). Análisis de los artículos publicados en revistas científicas". Revista latina de comunicación social, 2011, n. 66, pp. 101129.

http://www.revistalatinacs.org/11/art/926_Vicalvaro/05_ Nicolas.html

Masip, Pere. "Los efectos del efecto Aneca: análisis de la producción española en comunicación en el SSCl". En: Simposio AE-IC Investigar la comunicación en España, 2011. http://www.revistacomunicar.com/pdf/2011-04-Masip. $p d f$

Masip, Pere. "Efecto Aneca: producción española en comunicación en el Social science citation index. Anuario ThinkE$P I, 2011$, v. 5, pp. 206-210.

Perceval, José-María; Fornieles-Alcaraz, Javier. "Confucio contra Sócrates: la perversa relación entre la investigación y la acreditación". Anàlisi, 2008, n. 36, pp. 213-224.

http://www.raco.cat/index.php/analisi/article/viewFile/ $94533 / 119748$

Sabés, Fernando; Perceval, José-María. "Retos (y peligros) de las revistas científicas de comunicación en la era digital". En: I Congreso intl Latina de comunicación social, 2009, pp. 1-16. ISBN 9788499410012

http://www.revistalatinacs.org/09/Sociedad/actas/27 sabes.pdf

Stumpf, Ida. "Peer review in communication journals: viewpoint of editors, authors and referees". Perspectivas em ciência da informação, 2008, v. 13, n. 1, pp. 18-32.

\section{Anota en tu agenda: \\ $2^{\text {a }}$ Conferencia sobre calidad de revistas de ciencias sociales y humanidades (CRECS 2012) \\ Presentación del Anuario ThinkEPI, vol. 6 Valencia, 10 de mayo de 2012 http://www.thinkepi.net/crecs2012}

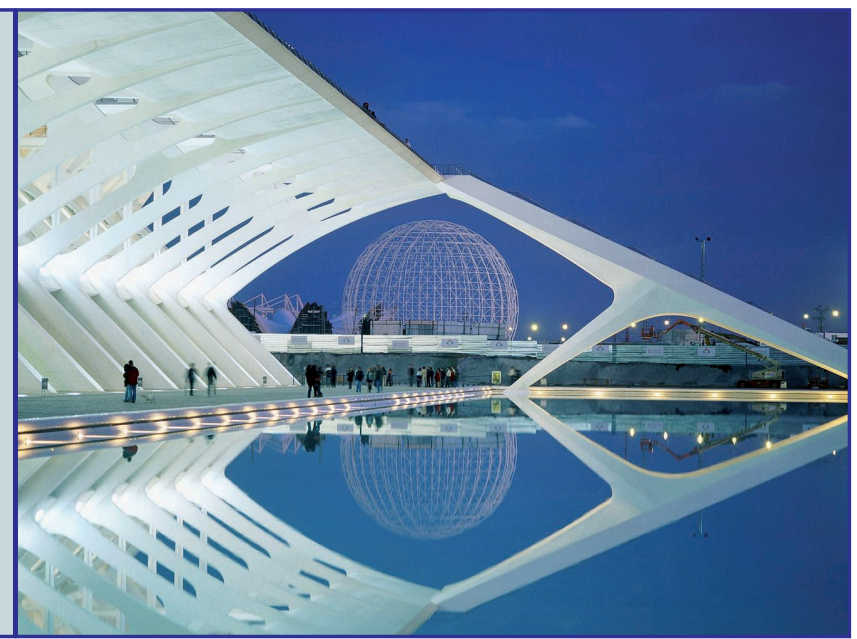

